\title{
Socioeconomics Inequality in Unhealthy Diet of Children in Sanandaj - Using Oaxaca Decomposition
}

\author{
Farideh Mostafavi ${ }^{1,}$; Ghobad Moradi ${ }^{\text {; }}$; Namamali Azadi ${ }^{2}$; Nader Esmaeilnasab ${ }^{\text {1; }}$ Maryam \\ Chamary ${ }^{3}$
}

${ }^{1}$ Department of Epidemiology and Biostatistics, Social Determinants of Health Research Center, Kurdistan University of Medical Sciences, Sanandaj, Iran

${ }^{2}$ Department of Epidemiology and Biostatistics, Iran University of Medical Sciences

${ }^{3}$ Tehran University of Medical Sciences

${ }^{*}$ Corresponding author: Farideh Mostafavi, Department of Epidemiology and Biostatistics, Social Determinants of Health Research Center, Kurdistan University of Medical Sciences, Pasdaran Ave, Sanandaj, Iran. Tel: +98-8733131366, E-mail: zakaria.pezeshki@gmail.com

Received: 22 Sep 2016

Accepted: 21 Nov 2016

Ppub: 23 Dec 2016

\begin{abstract}
Background: The present study intended to define socioeconomic inequalities of unhealthy diet among children aged 10-12 years using Concentration Index and Oaxaca decomposition method.

Methods: The present cross-sectional research studied 2506 children living in Sanandaj in 2015. The required data were collected using Food Frequency Questionnaire. The socioeconomic status (SES) was calculated by measuring household income through Principal Component Analysis technique. The inequality was measured using Concentration Index and the proportions of different determinants were defined using Oaxaca decomposition method.

Results: The results show that $50.75 \%$ of people had unhealthy diet (95\% CI: 48.79-52.71). The prevalence of unhealthy diet among people with high SES was lower than people with low SES (OR=0.41, 95\%CI: 0.30-0.57). Mother's high level of education was a protective factor against unhealthy diet $(\mathrm{OR}=0.55,95 \% \mathrm{CI}$ : 0.39-0.77). The concentration index for unhealthy diet was -0.14 (95\%CI: (-0.18)$(-0.09))$, which was indicative of concentration of unhealthy diet in the group with low SES $(\mathrm{p}<0.001)$. Oaxaca analysis showed the gap of prevalence of unhealthy diet in the poor and rich groups was $27.77 \%$ while $59.41 \%$ of the differences was because of the explained component. The highest contribution belonged to mother's education with $83.63 \%$ followed by place of residence with $17.81 \%$.

Conclusions: The results show that the prevalence of unhealthy diet was higher in people with low SES. Improving the awareness and literacy of mothers and performing interventions in living places can be effective in decreasing socioeconomic inequalities of unhealthy diet and prevention of chronic diseases in children.
\end{abstract}

Keywords: Unhealthy Diet; Socioeconomic Inequality; Concentration Index; Oaxaca Decomposition; Children 